\title{
Seminare 2014
}

\section{Praxiseröffnung/-übernahme}

Das Seminar richtet sich an Ärztinnen und Ärzte, welche vor einer Praxiseröffnung (Einzel-/Gruppenpraxis), dem Einstieg in eine Gruppenpraxis oder vor einer Praxisübernahme stehen.

\section{Themen}

- Juristische Aspekte (Praxisbewilligung, Zulassung zur Sozialversicherung, Vertragswesen)

- Gesellschaftsformen / Ehe- und Erbrecht (Privat-/Geschäftsvermögen, Güterstand, Erbschaftsplanung)

- Praxiseinrichtung (Inneneinrichtung, Kostenberechnung)

- Praxisadministration (Leistungserfassungsund Abrechnungssysteme)

- Bewertung einer Arztpraxis (Berechnung Inventarwert und Goodwill als Verhandlungsbasis)

- Finanzierung der Arztpraxis (Businessplan, Kredite, Absicherungsmöglichkeiten)

- Versicherungen/Vorsorge/Vermögen (Personen- und Sachversicherungen, Vorsorgeplanung).

\section{Sponsoren}

Die Kosten werden durch diverse Sponsoren (siehe www.fmhservices.ch) gedeckt.

\begin{tabular}{|c|c|c|}
\hline \multicolumn{3}{|c|}{ Daten } \\
\hline K01 & $\begin{array}{l}\text { Donnerstag, } \\
\text { 13. März } 2014 \\
9.00-16.30 \text { Uhr }\end{array}$ & $\begin{array}{l}\text { Zürich } \\
\text { FMT }\end{array}$ \\
\hline K02 & $\begin{array}{l}\text { Donnerstag, } \\
\text { 8. Mai } 2014 \\
\text { 16.00-20.30 Uhr }\end{array}$ & $\begin{array}{l}\text { St. Gallen } \\
\text { Hotel Einstein }\end{array}$ \\
\hline K03 & $\begin{array}{l}\text { Donnerstag, } \\
\text { 5. Juni } 2014 \\
9.00-16.30 \mathrm{Uhr}\end{array}$ & $\begin{array}{l}\text { Bern } \\
\text { Schmiedstube }\end{array}$ \\
\hline
\end{tabular}

\section{Praxisübergabe}

Das Seminar richtet sich an zukünftige Praxisübergeber/innen. Idealtermin: 5-10 Jahre vor geplanter Übergabe (aus steuer- und vorsorgeplanerischen Gründen).

\section{Themen}

- Juristische Aspekte (Praxisübergabevertrag, allg. Vertragswesen, Übergabe der Krankengeschichten)

- Nachfolgeplanung und Bewertung einer Arztpraxis (projektorientiertes Vorgehen in der Nachfolgeplanung, Berechnung Inventarwert und Goodwill als Verhandlungsbasis)

- Versicherungen/Vorsorge/Vermögen (Übergabe/Auflösung von Versicherungs- verträgen, Pensions- und Finanzplanung)

- Steuern (Steueraspekte bei der Praxisübergabe: Optimierung der steuerlichen Auswirkungen, Liquidations- und Grundstückgewinnsteuer, Bestimmung des optimalen Übergabezeitpunktes).

\section{Sponsoren}

Die Kosten werden durch diverse Sponsoren (siehe www.fmhservices.ch) gedeckt.

\begin{tabular}{|c|c|c|}
\hline \multicolumn{3}{|c|}{ Daten } \\
\hline K06 & $\begin{array}{l}\text { Donnerstag, } \\
\text { 20. März } 2014 \\
\text { 13.30-18.00 Uhr }\end{array}$ & $\begin{array}{l}\text { Zürich } \\
\text { FMT }\end{array}$ \\
\hline K07 & $\begin{array}{l}\text { Donnerstag, } \\
\text { 22. Mai } 2014 \\
\text { 16.00-20.30 Uhr }\end{array}$ & $\begin{array}{l}\text { St. Gallen } \\
\text { Hotel Einstein }\end{array}$ \\
\hline K08 & $\begin{array}{l}\text { Donnerstag, } \\
\text { 12. Juni } 2014 \\
\text { 13.30-18.00 Uhr }\end{array}$ & $\begin{array}{l}\text { Bern } \\
\text { Schmiedstube }\end{array}$ \\
\hline
\end{tabular}

\section{Finanz- und Steuerplanung}

Das Seminar richtet sich an Praxiseröffner/innen, Praxisübernehmer/innen sowie an bereits praxistätige Ärztinnen und Ärzte.

\section{Themen}

- Finanzplanung (Businessplan, buchhalterische Massnahmen vor Praxiseröffnung/ -übernahme, Standardkontenplan, doppelte Buchhaltung, EDV-unterstützte Buchführungslösung)

- Steuern (Steueraspekte bei Eintritt in die Selbständigkeit, Steuerfallen und Steuerrisiken, optimierte Steuerplanung).

\section{Kosten}

Für FMH Services-Mitglieder kostenlos.

\begin{tabular}{lll}
\multicolumn{2}{l}{ Daten } & \\
K11 & $\begin{array}{l}\text { Donnerstag, } \\
\text { 3. April 2014 } \\
\text { 13.30-18.00 Uhr }\end{array}$ & $\begin{array}{l}\text { Zürich } \\
\text { FMT }\end{array}$ \\
K12 & $\begin{array}{l}\text { Donnerstag, } \\
\text { 18. September 2014 } \\
\text { 13.30-18.00 Uhr }\end{array}$ & $\begin{array}{l}\text { Bern } \\
\text { Schmiedstube }\end{array}$ \\
\hline
\end{tabular}

\section{Praxiscomputerworkshop}

Der Workshop richtet sich an praxiseröffnende sowie an bereits praxistätige Ärztinnen und Ärzte.

\section{Inhalt}

- Anforderungen an ein Praxisinformationssystem (Einführung)

- Evaluationsprozess (projektorientiertes
Vorgehen in der Evaluation eines Praxisinformationssystems)

- Präsentation von sechs führenden Praxisinformationssystemen (Leistungserfassung, elektronisches Abrechnen unter Einbezug der TrustCenter, Agendaführung, Statistiken, Laborgeräteeinbindung, elektronische Krankengeschichte, Finanzbuchhaltungslösungen usw.).

\section{Kosten}

Für FMH Services-Mitglieder kostenlos.

\begin{tabular}{lll}
\multicolumn{2}{l}{$\begin{array}{l}\text { Daten } \\
\text { K13 }\end{array}$} & $\begin{array}{l}\text { Donnerstag, } \\
\text { 27. März 2014 } \\
\text { 13.30-18.00 Uhr }\end{array}$ \\
K14 & $\begin{array}{l}\text { Donnerstag, } \\
\text { Dërich } \\
\text { Technopark }\end{array}$ \\
& 13.30-18.00 Uhr 2014 & Bern \\
& BERNEXPO
\end{tabular}

Die Details zu den weiteren für Sie sehr informativen Seminaren:

$$
\begin{aligned}
\text { - Gruppenpraxis } \\
\text { - Röntgen in der Arztpraxis } \\
\text { - Praxismarketing für Ärzte } \\
\text { - Crashkurs Versicherungsmedizin } \\
\text { - Erfolgreich in die Pension } \\
\text { - Tarifwerk TARMED - Einführungskurs } \\
\text { - Telefonseminar für MPA } \\
\text { (bzw. Praxisteam) } \\
\text { - Kommunikation mit Patienten für MPAs } \\
\text { (bzw. Praxisteam) }
\end{aligned}
$$

\section{entnehmen Sie bitte ab unserer Web-} site www.fmhservices.ch $\rightarrow$ Seminare.

\section{Anmeldung und Auskunft}

www.fmhservices.ch oder FMH Consulting Services, Cornelia Fuchs, Burghöhe 1, 6208 Oberkirch, Tel. 04192500 77, Fax 0419210586.

\section{Hinweis}

Bei sämtlichen Seminaren, bei denen die Kosten teilweise oder gänzlich von Seminarsponsoren gedeckt werden, werden die Teilnehmeradressen den jeweiligen Sponsoren zur Verfügung gestellt.

\section{Annullierungsbedingungen}

Bei Abmeldungen oder Fernbleiben werden folgende Unkostenbeiträge erhoben:

- 50 CHF pro Person ab 14 Tage vor Seminarbeginn;

- 100 CHF pro Person ab 7 Tage vor Seminarbeginn oder Fernbleiben. 